\title{
RENÉ THOM ET LA RÉHABILITATION DES FORMES SUBSTANTIELLES
}

\author{
Morier Clément ${ }^{1}$ \\ (Faculté de Droit, Université Jean Moulin Lyon III) \\ Bruno Pinchard ${ }^{2}$ \\ (Directeur de l'école doctorale de philosophie de la \\ région Rhone-Alpes-France)
}

\section{Introduction}

Réfléchir philosophiquement sur la notion de nature aujourd'hui nous oriente vers l'œuvre d'un des penseurs majeurs du $\mathrm{XX}^{\mathrm{e}}$ siècle français, pour qui la forme est le maître mot. Le trait spécifique de la pensée de René Thom est de tenir ensemble science et philosophie pour interroger les propriétés géométriques des morphologies naturelles. Notre étude soulève la question suivante : pouvons-nous redécouvrir les modalités philosophiques de sa pensée des formes, afin de poser à nouveau frais la fécondité d'un savoir qualitatif de la nature pour notre temps ? L'hypothèse de ce travail vise à établir qu'une réhabilitation de la notion de forme substantielle est le creuset d'un questionnement actuel sur le concept de nature, à rebours du tournant réductionniste de la mécanique cartésienne et de ses prolongements contemporains.

D'abord, nous tâcherons de montrer que l'évènement représenté par l'entrée de Thom en philosophie a eu un précédent central inscrit au cœur de l'œuvre de Leibniz. Cette mise au point effectuée, nous serons à même d'interroger les enjeux du néo-aristotélisme thomien, dans l'appréhension

\footnotetext{
${ }^{1}$ clementmorier@yahoo.fr

2 pinchard.bruno@gmail.com
}

Philosophica, 47, Lisboa, 2016, pp. 125-140. 
scientifique de la nature. Nous présenterons ses grands gestes intellectuels, dont nous tenterons d'évaluer la signification non seulement philosophique, mais proprement métaphysique. Entendons par là un discours qui essaie de définir les formes pures qui gouvernent le monde naturel : il ne serait pas simplement le développement d'une suite de chocs, mais ce monde, en se déployant précisément, développerait des germes qui euxmêmes répondraient à des formes primordiales. En ce sens, les métaphysiciens tels Thom recherchent des principes, c'est-à-dire la façon dont le réel répond à des déploiements de concentration d'énergie et de puissance informative initiales gouvernant les développements temporels.

$\mathrm{Au}$ terme de quoi, nous aurons une vue suffisamment organisée sur la réhabilitation thomienne des formes substantielles, pour illustrer la nécessité de restituer à la nature un de ses concepts fondateurs.

\section{Leibniz ou l'histoire d'une erreur mémorable de M. Descartes}

L'œuvre de Leibniz nous permet de saisir dans quels champs les dispositifs intellectuels de Thom se présentent. La révolution scientifique constituée par l'œuvre de Descartes et de Galilée a consisté à mesurer le réel à partir d'une conception mécanique de la matière : en le réduisant à des chocs de quantités maitrisables par les mathématiques, des séries numériques permettent de paramétrer l'évolution de leurs trajectoires. Ce n'est pas seulement la virtuosité de l'Occident en mathématiques qui s'exprime dans ces victoires, c'est véritablement une explosion du monde naturel connu par les hommes jusque-là. Ce qui sombre dans cet évènement est l'appréhension magique du monde des influences : l'idée que par un certain nombre de gestes, de paroles et de conceptions techniques, les hommes peuvent agir à distance sur les objets. Or, l'évènement écrasant de cette révolution démontre que désormais, le contact entre les objets ne dispose d'aucun moteur ou matrice spirituelle et explique seul la métamorphose, réduite à une question d'enchainement de forces. Tout le siècle ayant précédé cet évènement s'est vu sombré dans la découverte géniale de ces deux hommes. Prend fin le siècle de la Renaissance, qui est toute d'astrologie, de magies amoureuses, de conceptions vivantes de la nature et de fluidité des formes répondant à la main créatrice d'artistes, qui ne disposent pas d'une loi mathématique, mais obéissent à une tradition qui les précèdent et dans laquelle ils innovent. Ce monde que Giordano Bruno, Marcil Ficin ou Rabelais incarnaient, en un instant, par la seule puissance du premier livre de Descartes, les règles pour la direction de 
l'esprit ${ }^{3}$, s'est effondré. A commencé un monde qui certes aujourd'hui a pris la forme du contrôle informatique, mais elle est elle-même la fille de ce que Descartes dès sa première heure, a nommé de façon prémonitoire une mathesis generalis ou universalis : un savoir qui établit dans l'espace un contrôle de tous les mouvements. Seuls quatre penseurs furent véritablement capables d'entendre la nouveauté de ces découvertes : Pascal, Spinoza, Malebranche et Leibniz.

Ce dernier écrira un premier discours d'une perfection surprenante, Le discours de métaphysique ${ }^{4}$, dans lequel il dresse à sa façon le bilan de cette nouvelle philosophie. Or, ses paragraphes 10 et 11 engagent ce que Thom répètera en son temps. Leibniz enregistre avec brio la toute puissance des explications du mécanisme, étant le mathématicien ayant su d'emblée identifier une faute dans l'équation du mouvement de Descartes ${ }^{5}$. Il reprit le mécanisme du choc qui comprenait la séquence de rencontre et d'interaction des corps analysée par cet auteur et l'équation qu'il en avait tiré, mais Descartes n'avait pas enregistré un autre phénomène faisant partie intégrante des chocs. Il s'agit du ressort des corps dans leurs interactions : l'énergie emmagasinée dans l'interaction - appelée en physique l'action - est le véritable moteur de la propulsion.

Leibniz comprenait l'enjeu exact de ces découvertes parce que, précisément, il en avait une maitrise tellement admirable qu'il va les révolutionner en proposant de passer d'une mécanique simple à une dynamique. La dynamique est cette théorie mathématisable, grâce à son invention du calcul différentiel, permettant non seulement de paramétrer les effets de ressort qu'une entité subie lorsqu'elle est compressée dans un choc, mais aussi d'enregistrer une plus grande précision dans le calcul des trajectoires. Le calcul différentiel signera certes la supériorité de Leibniz sur le mécanisme cartésien, mais, loin d'être aveugle, il se rend bien compte que le changement du monde qui vient de s'effectuer est irréversible. Il n'a nullement l'intention de modifier le concept de mathesis universalis. Le monde a perdu ses influences, ses qualités et ses illusions de pouvoir introduire des sentiments dans les choses. Les destins humains évolueront désormais dans un monde de trajectoires, se calculeront et se

Descartes, Règles pour la direction de l'esprit, (trad. J. Sirven), Paris : Vrin, 1997.

4 Leibniz, Discours de métaphysique et correspondance avec Arnauld, (trad. G. Le Roy), Paris : Vrin, 1993.

Voir la courte démonstration traduite en septembre 1686 dans la revue Nouvelles de la République des Lettres. Texte consulté en ligne le 01 février 2016 sur le lien : http:// www.philo-bernard.fr/images/QFV/QFV_sep1686.pdf 
modéliseront à partir des algorithmes mathématiques disponibles pour la communauté scientifique.

Cependant, Descartes faisait une erreur. Leibniz formulera alors une remarque, capitale pour l'histoire de Thom. Ce qu'il manque au cartésianisme, souligne-t-il, est la considération du fait suivant : si nous voulons que deux corps soient dans un effet de choc et de ressort, il faut que ces corps disposent d'une solidité et d'une structure. S'ils ne veulent pas voler en éclat et se pulvériser mais emmagasiner l'énergie du choc et la renvoyer, les corps doivent avoir une consistance, afin que le monde ne soit pas simplement un amas de poussière issu de ces frottements - depuis le temps que les chocs s'effectuaient, le monde aurait poudroyé.

Leibniz pose alors la question de savoir ce qui fait la consistance des corps. Est-ce que le monde est un agrégat, une pulvérulence, dont seul l'atomisme serait le moteur profond et l'organisateur? Les lois de ressort montrent bien que les corps ont des forces de cohésion d'une tout autre réalité que le simple atomisme, n'expliquant rien de leur énergétique. Admettons même que le fracas des pierres ne soit qu'une pulvérulence qui s'entrechoque selon des lois de cohésion hasardeuse, de toute façon, les corps vivants et leurs interactions biologiques ne peuvent répondre à des lois simplement mécaniques. Il est impératif de restituer l'unité de l'organisme pour ne pas le réduire à un bricolage futuriste de pièces extérieures les unes aux autres.

Or, le geste philosophique leibnizien concède à la physique une explication mécaniste du monde, mais pose comme fondamentalement nécessaire d'ajouter sur un plan métaphysique des germes ou principes, unificateurs des formes et des phénomènes qui apparaissent dans le monde. Leibniz donnera plusieurs noms à cette entité organisatrice, ayant pour fonction principale de conférer de l'unité au réel, c'est-à-dire de sauver les entités d'une pure pluie d'atomes et faire en sorte que le monde naturel ait un visage organique. Il élaborera des hypothèses nombreuses mais toute convergentes, dont nous retiendrons ici quatre aspects.

Leibniz va s'appuyer sur l'intuition des Renaissants, foudroyée par le système cartésien alors qu'ils touchaient l'idée d'un principe vivant derrière le mécanisme des corps. Cette juste intuition métaphysique les faisait échouer dans leur physique, car ne disposant pas des mathématiques permettant de sérier l'état de la matière, ils introduisaient des principes métaphysiques dans une explication de la physique qui trouvait là son moment magique. Leibniz choisit comme exemple Paracelse, magicien de la Renaissance, et alchimiste parce que médecin, persuadé que les corps obéissaient à des archés, notions que Leibniz souhaite préserver. 
Lui, mathématicien et rationaliste absolu, puise dans l'ésotérisme occidenta $^{6}$ et l'alchimie des notions purement magiques pour les introduire dans son système. Derrière une explication intégralement mécanique du monde évolueraient des archés organisatrices.

Leibniz remonte à des suppositions plus archaïques encore, issues de l'Antiquité égyptienne, autour de principes organisateurs présents dans la tradition hermétique et néoplatonicienne, dont le principal représentant était Plotin. Ce dernier supposait une nature gouvernée par une suite d'entités spirituelles réparties entre l'Aphrodite céleste et l'Aphrodite terrestre'. La nature ne serait pas simplement un paquet d'atomes démocritéens, mais suivrait des processus de développement, figurables telle une maternité divine - l'Aphrodite terrestre, sorte d'organisation vivante ou de sexuation nourricière des interactions sur la surface de la Terre - ellemême gouvernée par un principe plus élevé procédant de l'Unité pure gouvernant toute manifestation, l'Aphrodite céleste. Leibniz reprend cette conception à partir de la notion d' «âme » : les interactions expliquées par la physique suivant des séries mathématisables, sont en réalité gouvernées selon une psychophysique par des principes énergétiques qui sont des âmes, foncteurs organisateurs de ces interactions.

Un troisième versant de cette enquête trouvera dans l'œuvre de Thomas d'Aquin un principe organisateur et unifiant identifié par Aristote lui-même, qui protégeait les corps des déformations et des dislocations, et renommé forma substantialis. Leibniz dégage ce concept pour le réinvestir dans son dessein, celui de sauver le monde moderne de la pure violence des conflits aveugles mécaniques, gouvernés selon lui par des régimes spirituels, concentrés dans des points ou formes qui sont les moteurs réels du développement de la nature.

Dans un dernier versant enfin, ayant découvert que les interactions entre les corps dépendaient d'une quantité d'action reposant sur la force qu'un corps accumulait en lui, Leibniz soupçonna que cette force n'était pas simplement un ensemble de vibrations matérielles étant comme emmagasinées dans les corps, mais que cette force disposait d'une spontanéité. Ce phénomène de la force ne semble pas se réduire à un processus de frottement des corps, mais appartiendrait plutôt à ce même plan métaphysique : archès, âmes, forme substantielle, force, voila le quadruple visage dégagé par le philosophe pour refonder et la science et la philosophie de son temps.

6 Voir Antoine Faivre, Accès de l'ésotérisme occidental, Paris : Gallimard, 1986.

7 Voir Plotin, Ennéades, Tome 3, (trad. E. Bréhier), Paris : Les Belles Lettres, 1995. 
Dans les paragraphes 10 et 11 du Discours de métaphysique, il se rend compte, lui le savant, qu'il se ridiculise et prend des risques philosophiques extrêmes. Susceptible de perdre son sérieux en faisant rentrer ces points substantiels dans le mécanisme, il souligne toutefois : « Je sais que j'avance un grand paradoxe en prétendant de réhabiliter en quelque façon l'ancienne philosophie », celle de la Renaissance, du néoplatonisme alexandrin et de l'aristotélisme de Saint-Thomas, " et de rappeler post liminio ", c'est à dire par delà l'échelle de la mort où Descartes avait condamné « les formes substantielles presque bannies $»^{8}$. Il n'avait laissé aux philosophes que le choix de les renier pour travailler les interactions physiques, ou de régresser avec les anciens et leurs formes, qu'il nommait «qualités occultes » par une extrême moquerie pour celui qui mettait ses valeurs dans le clair et le distinct.

Mais peut être qu'on ne me condamnera pas légèrement, quand on saura que j'ai assez médité sur la philosophie moderne, que j'ai donné bien du temps aux expériences de physique et aux démonstrations de géométrie?.

Ayant montré l'erreur mémorable de Descartes, Leibniz s'était « longtemps persuadé de la vanité de ces êtres $»^{10}$. La réintroduction des formes dans la modernité lui semblait initialement une activité dérisoire, mais il ajoute :

que j'ai été enfin obligé de reprendre malgré moi et comme par force, après avoir fait moi-même des recherches qui m'ont fait assez reconnaitre que nos modernes ne rendent pas assez de justice à saint Thomas et à d'autres grands hommes de ce temps-là et qu'il y a dans les sentiments des philosophes et théologiens scolastiques bien plus de solidité qu'on ne l'imagine, pourvu qu'on s'en serve à propos et en leur lieu ${ }^{11}$.

Leibniz, sans prétendre expliquer à nouveau la physique par les formes substantielles, souligne en revanche l'impossibilité de comprendre la structure fine et le caractère consistant des corps naturels sans faire appel à ces archès, ou formes. Il produit là un geste philosophique si mémorable qu'il sera à l'origine d'une école ayant trouvé en Goethe un disciple fidèle, non seulement par ses travaux morphologiques sur la lumière, les plantes

8 Leibniz, Discours de métaphysique et correspondance avec Arnauld, p. 46.

9 Ibid.

10 Ibid.

11 Ibid. 
et la botanique ${ }^{12}$, mais surtout par sa doctrine de l'«Urphänomen » : de tels phénomènes organisateurs seraient à l'origine du déploiement d'une plante ou d'un embryon selon une loi de structure. Ses spéculations appartiennent toutes à un regard et une visée morphologique, appréhendant et engageant la pensée dans les formes.

\section{L'écho de la querelle leibnizienne dans les audaces scientifiques de Thom}

Le Discours de métaphysique reste déterminant dans le milieu de Thom, où nul n'entrait sans avoir mesuré qu'il recommençait une même critique de la modernité en réhabilitant ce dispositif. Il se dit morphologue à une époque - fin des années 1960 en France - où ce n'est plus la révolution mécanique mais trois sciences au moins, qui prétendent en avoir fini avec la pensée goethéo-aristotélicienne des formes. Ce géomètre de Montbéliard entre en scène en $1972^{13}$, alors que ce bilan semble irréversible tant pour la thermodynamique, la mécanique quantique que le formalisme linguistique, et artistique.

La thermodynamique définit soit des systèmes clos, pour lesquels règnent des lois de dépérissement et de dissipation de l'énergie conduisant la différenciation du monde à l'usure, et en regard de quoi rien ne sert de maintenir des archès organisatrices ; soit des systèmes ouverts, où les déperditions donnent lieu non pas à des fixations de formes, mais au contraire à des phénomènes d'émergence ${ }^{14}$. Sur un mode aléatoire ces systèmes peuvent engendrer, à force d'itérations, des manifestations plus stables qui deviennent phénoménologiquement des formes, mais, sur le plan de la construction de l'objet, ne sont que des stabilisations provisoires ne répondant pas à un Urphänomen.

La mécanique quantique travaille des quantités infinitésimales tellement fines que les mises en forme ne comptent pas dans ses espaces. Le monde naturel se réduit à une pelote d'épingles où les entités, traversables et sans plus aucune consistance organique, résultent elles-mêmes d'échanges entre charges énergétiques selon des lois qui, en outre, ne sont

12 Voir Jean Petitot, Morphologie et esthétique, La forme et le Sens chez Goethe, Lessing, Lévi-Strauss, Kant, Valéry, Husserl, Éco, Proust, Stendhal, Paris : Maisonneuve et Larose, 2004.

13 René Thom, Stabilité structurelle et morphogenèse, Paris : Interéditions, 1972.

14 Voir Ilya Prigogine, Isabelle Stengers, La nouvelle Alliance. Métamorphose de la science, Paris : Folio, 1986. 
même pas fixes, car fondées sur l'incertitude majeure de leurs conditions initiales ${ }^{15}$. Que ce soit par le probabilisme ou le calcul statistique des interactions, la physique quantique engendre au plan philosophique un phénoménisme de type bouddhique, où le monde est une maya faite de nuages de points et de consistances provisoires qui apparaissent certes phénoménologiquement, mais n'existent pas dans le vrai plan où le réel évolue.

Un troisième plan montre que les formalismes développés avec Hilbert ne se cantonnent pas aux objets mathématiques, mais se déploient dans le domaine de la linguistique avec Wittgenstein ${ }^{16}$, de la musique avec Schoenberg ${ }^{17}$, ou encore de la peinture avec Kandinsky ${ }^{18}$. Puisqu'il n'y a pas de fondement ou de substance dans le monde naturel, ses manifestations résultent d'opérations locales entre des séries d'évènements discontinus. Selon une perspective constructiviste, l'enjeu est de créer des systèmes purement formels reposant sur des axiomatiques arbitraires. Sur le modèle des espaces de Hilbert, ces opérativités axiomatisables substituent un sérialisme à toutes formes stables. Ce formalisme véhicule une explication de la nature totalement basée sur l'agrégat, que nous pouvons surveiller et contrôler intégralement, puisque l'agrégat est comptable par informatique, outil de traçabilité de toute évènementialité dans cette nouvelle maya.

Thom n'avait aucune vocation à produire un évènement quelconque parmi toutes ces conceptions, si ce n'est que, comme Leibniz il était doué d'une virtuosité supérieure en mathématiques, lui permettant de produire une critique mathématique de l'idéologie de son temps. Thom fait carrière dans une science locale coupée de ces grands enjeux, mais, en travaillant à partir de certaines surfaces, cette science topologique en développement permettait d'obtenir des théorèmes non réductibles à leur seul examen algébrisé, et supposait un type d'explication et de mathesis distincts de l'explication strictement quantitative. Après avoir été décoré de la médaille Fields en 1958, Thom développera la " théorie des catastrophes » (TC), dans le but de reprendre ces espaces topologiques afin d'envisager une nouvelle interrogation sur les fondements des morphologies sensibles. On dit que la nature n'est qu'une suite de chocs ou d'interactions moléculaires : et si, l'embryon dans les phases de son développement obéissait à

15 Voir Michel Bitbol, Mécanique quantique. Une introduction philosophique, Paris : Flammarion, 1996.

16 Ludwig Wittgenstein, Tractatus logico-philosophicus, (trad. G-G. Granger), Paris : Gallimard, 2001.

17 Arnold Schoenberg, Traité d'harmonie, (Trad. G. Gubisch), Paris : Médiamusique, 2008.

18 Voir Michel Henry, Sur Kandinsky, Paris : PUF, 2005. 
des systèmes de formes, que l'on peut écrire et calculer, représentant une intelligibilité formelle du développement cellulaire de l'embryogenèse ${ }^{19}$ ? Et si, quand l'eau vient à bouillir, dans le moment d'agitation où les premières bulles remontent du frisson annonçant l'ébullition, il ne s'agissait pas simplement d'un réchauffement de la matière, mais qu'il apparaissait des géométries de cette transition de phase que représente le passage à l'ébullition, ou à la glaciation, transition de phase de la cristallisation ? $E t s i$, dans le langage, les relations entre les sujets et les verbes n'étaient pas simplement des « actes" de performativité basés sur des « jeux », mais reposaient sur une mimésis par laquelle la langue répète ce que fait la nature elle-même ? Dans la phrase « le chat mange la souris » les deux phénomènes, l'articulation linguistique et l'articulation naturelle du bond du chat, obéissent selon Thom à une même topologie, nommé « le lacet de prédation $»^{20}$.

Ce dispositif dresse une contre-offensive contre la mathesis des modernes, tant les champs de l'aplatissement réductionniste de la modernité se trouvent tous défiés par des archès non pas alchimiques, mais des formes substantielles topologiques, dont la communauté scientifique dispose de l'algébrisation. Le caractère polémique que ces actions intellectuelles supposent ne doit toutefois pas masquer les objections réelles qu'elles véhiculent. En effet, puisqu'il y a une générativité de la topologie algébrique et qu'elle répond à des lois mathématiques, si Thom réintroduit des entités topologiques comme semences organisatrices du réel en critiquant une mathesis aveugle à de telles entités, ne pourrions-nous soupçonner qu'en réalité il ne fait qu'étendre l'empire de la mathesis? Thom jouirait d'une puissance de science telle que son aspect faustien serait en mesure de produire une équation vérifiable des archès fondatrices, et de conquérir par les mathématiques des objets laissés jusqu'ici au secret des âmes ou de la nature elle-même.

A cette idée d'un Thom tyrannique, il fait une objection et propose une voie décisive en démontrant la fécondité d'une seconde mathesis, distincte du contrôle intégral des interactions où domine la valeur de prédictibilité, malgré son caractère aléatoire dans le probabilisme quantique. Laplace envisage encore le déterminisme comme absolu, mais, bien que des béances soient admises dans ses lois à partir de Cournot, et qu'un penseur comme Prigogine le rejette ontologiquement, les mathématiques restent une science prédictive qui anticipe le temps dans son équation. En revanche,

19 René Thom, Modèles mathématiques de la morphogenèse, Paris : C. Bourgeois, 1974.

20 Ibid. 
Thom souligne que les formants géométriques de sa topologie ne donnent pas accès à une prédictibilité. Ses formes qualitatives acceptent des échappées et des incertitudes sans avoir un pouvoir de gouvernance absolu sur le développement énergétique de la matière. Elles prêtent dans ce cas le flanc à une critique opposée : cette œuvre topologique, par son rattachement au domaine du qualitatif, perd-elle son sérieux mathématique en devenant finalement herméneutique, voir poétique ? Thom répondait que « prédire n'est pas expliquer $»^{21}$. Il tentait de créer des horizons d'attente et des attracteurs qualitatifs pour de l'évènement, à partir desquels esquisser les concentrations de matière sur certains points, sans disposer dans cette écriture-là d'une prédictibilité déterministe. Tout en maintenant cette position, il soutenait le déterminisme contre ceux qui au nom des découvertes quantiques, prônaient son remplacement par des émergences aléatoires. Il défendait l'idée que les savoirs déterministes sont effectivement prédictifs, mais peuvent obéir à une loi de structure topologique dans leur déploiement phénoménal, se tenant dans l'horizon d'une explication morphologique. Il existait donc une domination d'un autre genre des mathématiques, non pas une explication en termes de fonctions de trajectoires, mais en termes de diffusion, d'attraction et de frontières par lesquelles un objet se borde et voie évoluer ses formes dans l'espace.

En dégageant cette voie qualitative, les crédos de la philosophie moderne devenaient problématiques : fallait-il renoncer à la réduction de la philosophie à des jeux de langage wittgensteiniens, à la déconstruction ou destruction heideggérienne de la métaphysique pour envisager sa refondation topologique, et abandonner le mot d'ordre de Derrida visant à défaire les bords pour déconstruire les formes naturelles et sémantiques? Dans l'opposition expliquer / prédire se dégage un plan à travailler dans lequel les entités ne résultent pas tant du nihilisme, mais offrent une voie objective d'étude de la nature dépassant l'opposition entre science quantitative et phénoménologie qualitative. En effet, Thom évolue dans ces années où la phénoménologie domine et l'arc reliant Husserl à Merleau-Ponty médite sur la crise galiléenne dans les sciences européennes. Husserl avait tenté de reconstruire par la phénoménologie l'aspect qualitatif du monde pulvérisé dans la révolution galileo-cartésienne, afin de refonder une communauté d'esprit basée les vécus de la conscience, organisés en systèmes de nature transcendantale ne dépendant d'aucune manière des interactions physiques. Les phénoménologues dégagent

21 René Thom, Prédire n'est pas expliquer, Paris : Flammarion, 1991. 
des qualités douées de reconnaissance réciproque entre les êtres du monde, à partir d'un "Dasein » chez Heidegger, d'une « chair » chez Merleau-Ponty ou d'un « visage » chez Lévinas.

Or, leurs analyses dépendaient du vécu de phénomènes herméneutiques reposant sur la conscience et les intentions d'une subjectivité, douée d'intériorité spirituelle. Mais, Thom montrait qu'ils ne reposaient pas tant sur une subjectivité que sur des lois de l'espace. Ce que les phénoménologues attribuent aux vécus "pathétiques " des expériences de la conscience de soi, selon Michel Henry, ou aux vulnérabilités lévinassiennes d'une conscience déportée dans son altérité, loin d'être le comble de la subjectivité occidentale, appartient selon Thom à des plicatures de l'espace, à des lois de générativité des points, donc à une objectivité. La phénoménologie, accompagnant le relativisme du formalisme quantique, faisait l'épochè de l'existence d'une nature, déployée selon des formes substantielles. Ainsi, une voie philosophique a «narcissisé » et " pathétisé » la culture dans des états d'âme en « subjectivisant» la nature, alors qu'elle demandait à être reconnue à partir des lignes de forces organisationnelles de sa manifestation, supposant ainsi un réalisme physique du monde signifiant, selon des formes douées de potentialisation déterminante sur les rapports de l'égo à lui-même.

Contrairement à ce que la tradition cartésienne suppose, le fondement n'est pas l'égo mais la frontière, la singularité ou catastrophe qui partage les espaces. L'irruption structurante du fini par différenciation morphologique est la condition première d'une intelligibilité. Le sens apparait sur l'arrête, lors du déchirement qui fulgure dans l'espace. L'ensemble de la TC n'est en cela rien d'autre que le développement de ce déchirement initial. C'est pourquoi, la proposition la plus vraie qui ait jamais été formulée est la suivante : "è entéléchéia chôrizei », l'acte sépare selon Aristote $^{22}$. Une différence originelle dans le continu produit un évènement de séparation, et toute organisation structurée morphologiquement en est un dérivé, par déploiement du point ou germe organisationnel, des motifs de la topologie thomienne.

22 Aristote, Métaphysique, Z 13, 1039 a 6-7, cité in René Thom, Esquisse d'une sémiophysique : Physique aristotélicienne et Théorie des Catastrophes, Paris : Interéditions, 1988, p. 155 et p. 227. 


\section{Sémiophysique ou physique de l'ousia?}

Les enjeux thomiens de cette étude qualitative de la nature s'éclairent par une lecture complémentaire d'Aristote. Devant l'urgence d'abandonner les systèmes présocratiques et ses conceptions figées dans une forme de sacralité, Aristote se demande à quelle condition l'homme peut investir philosophiquement la nature : comment un point, une ligne, une surface seraient-il vivants ${ }^{23}$ ? Le formalisme pythagoricien appréhende l'organisation mathématique du réel, mais comment penser le mouvement et la vie selon les bords figés de la géométrie euclidienne ? Aristote examine plusieurs apories : soit le mouvement n'existe pas, soit la nature est le reflet d'un monde spirituel caché dans l'invisible selon des conceptions mystiques ou orphiques. Aristote répond en posant la nécessité de concevoir, au centre de l'évènement naturel, une réalité nouvelle qu'il nomme «ousia» ou substance. Les entités naturelles ne sont pas simplement une maya ou une fantasmagorie d'images platoniciennes, mais sont séparables et closes sur elles-mêmes. Nous pouvons les désigner comme telles selon la séparation du fond sur lequel elles apparaissent, «tode ti choriston » : la séparabilité fait l'unité du phénomène réel, dynamique et unifié de la substance dont la vie est formée ${ }^{24}$.

Or, le noyau de la substance, sa puissance de support faisant qu'elle n'est pas simplement un point ou un plan pythagoricien, est son substrat, niche matricielle de la vie dans laquelle chaque entité naturelle repose. La main du mathématicien se distingue de celle du physicien dans la mesure où le premier, dans la substance voit juste sa forme, tandis que le second comprend la forme en tant qu'elle est engagée dans un substrat. «Ont une nature tous les étants qui possèdent en eux un principe de ce genre [de mouvement]. Et ces étants sont tous des substances », douées de vitalité par le déploiement du mouvement qui les habite, « car ce sont des sortes de substrats, et la nature est toujours dans un substrat $»^{25}$. Elle n'est pas un système idéal, mais une forme engagée dans une réalité qui la contient, la clos sur elle-même et en fait une entité séparable. Thom n'a jamais conçu les formes simplement comme telles, sinon à être victime d'un pythagorisme incapable de porter la vie. Il considérait les formes comme un en-

23 Voir le livre M dans Aristote, Métaphysique, Tome 2, Livres H-M, (trad. J. Tricot), Paris : Vrin, 2004.

24 Voir le Livre Z dans Aristote, Métaphysique, Tome 1, Livres A-Z, (trad. J. Tricot), Paris : Vrin, 1991.

25 Aristote, Physique, livre II, 1, 192b-193a, (trad. P. Pellegrin), Paris : Flammarion, 2002. 
semble de singularités qualitatives dans un substrat. La forme thomienne n'est jamais indépendante du milieu énergétique - ou système dynamique - dans lequel elle se déploie. En effet, sa géométrie dynamique contient un mouvement de métamorphose se manifestant non pas dans le plan euclidien neutre, mais dans des systèmes dynamiques substrats ${ }^{26}$ : la fluence de la forme se déploie dans une continuité évolutive, à partir de la transformation des systèmes dynamiques. Sa topologie se constitue en une suite d'interactions entre la forme et son substrat, selon une diffusion de l'une dans l'autre et une réactivité de celui-ci à celle-là.

Comment dès lors fournir un concept pour organiser ces interactions, et répondre au dessein d'Aristote d'approfondir la nature par ce caractère matriciel et réceptif qu'est le substrat ? Le choix d'une conceptualité similaire entre Aristote et Thom signe leur convergence pour vaincre le platonisme : c'est le bord, ou la limite, qui décide de la façon dont une forme s'envoie dans son substrat. L'ensemble des limites qui entourent le corps est dans un rapport strict avec l'axe organisateur de l'entité, et permet d'étudier la façon dont une forme vie sa motricité dans l'espace.

limite se dit de l'extrémité d'une chose c'est-à-dire du premier point au-delà duquel il n'est plus possible de rien appréhender de la chose, et du premier point en deçà duquel est son tout. - c'est aussi la forme [...]. La limite est aussi la substance formelle de chaque chose et sa quiddité ${ }^{27}$.

L'axe qui organise le corps vitalise le bord de l'entité substrat dans laquelle il s'engage totalement, au point que lors d'une perturbation, la sensibilité répandue aux limites vient à réagir en étant aussi importante que son centre. Géométrie du vivant, la TC se focalise sur les différentes modalités topologiques de ce moment précis - la catastrophe - où l'axe organisateur de l'entité étudiée - nommé un puits de potentiel - reçoit une perturbation sur ses limites qu'il ne peut stabiliser. Dans cette crise, l'entité entre en métamorphose en modifiant son mode de viabilité par un changement brusque d'état stable, réponse aux impulsions de déséquilibre qui surviennent dans un système dynamique et qui déploient la croissance organisationnelle d'une entité naturelle quand elles ne la détruisent pas. Ces sollicitations à la métamorphosent engagent les formes dans un développement évolutif vers des états supérieurs de leur organisation.

26 Voir Claude Paul Bruter, Topologie et perception, Tome 1, Paris : Maloine, 1974.

27 Aristote, Métaphysique, Tome 1, Livre $\Delta, 17$, p. 205. 
Par cet accent posé sur les limites, et la progression de leur complexité morphologique initiée à la suite d'une série de bifurcations enrichissantes, Thom libère l'étude de la nature du pur formalisme. Il interroge les substances selon l'habitation progressive de leur espace par ses limites, suivant des phénomènes de réactivité énergétique sur les bords, soumis aux complications topologiques impulsées par les ébranlements que l'entité subie. Il oriente ainsi l'analyse sur les modalités de transformations stables des systèmes dynamiques, plongés dans une dimension d'évolution, afin de travailler le profil qualitatif de leur mutation. Rétractions pulsatiles, vibrativité continue d'un substrat en variation, potentialité de métamorphose, bref Thom fournit par son néo-aristotélisme une cartographie des limites dotées de puissance de création et de mutation de formes. Loin de forcer le réel en l'obligeant à entrer dans des triangles et des boules, il s'agit au contraire de créer des entités topologiques qui restituent aux formes naturelles leurs sensibilités internes, et qui les protègent d'un jugement sommaire issu de la science contemporaine. Au jugement essentiellement réductionniste produit par la logique néo-libérale autant que néo-darwiniste, ne voulant que des coups, des chocs, des quantifications et des sélections, Thom nous intime non de revenir à un créationnisme, mais de chercher par les mathématiques modernes les rétractivités et les déploiements, les invaginations et les prolongements, autant de dispositifs restituant le travail morphologique proprement substantiel et herméneutique de la limite. 


\section{BIBLIOGRAPHIE}

Aristote, Métaphysique, Tome 1, Livres A-Z, (trad. J. Tricot), Paris : Vrin, 1991.

— Métaphysique, Tome 2, Livres H-M, (trad. J. Tricot), Paris : Vrin, 2004.

Bitbol, Michel, Mécanique quantique. Une introduction philosophique, Paris : Flammarion, 1996.

Bruter, Claude Paul, Topologie et perception, Tome 1, Paris : Maloine, 1974.

Descartes, René, Règles pour la direction de l'esprit, (trad. J. Sirven), Paris : Vrin, 1997.

Faivre, Antoine, Accès de l'ésotérisme occidental, Paris : Gallimard, 1980.

Henry, Michel, Sur Kandinsky, Paris : PUF, 2005.

Leibniz, Gottfried Wilhelm, « Démonstration courte d'une erreur considérable de M. Descartes », Nouvelles de la République des Lettres, s.1., 1686, 9991003. Texte disponible en ligne sur le lien : http://www.philo-bernard.fr/ images/QFV/QFV_sep1686.pdf

- Gottfried Wilhelm, Discours de métaphysique et correspondance avec Arnauld, (trad. G. Le Roy), Paris : Vrin, 1993.

Petitot, Jean, Morphologie et esthétique, La forme et le Sens chez Goethe, Lessing, Lévi-Strauss, Kant, Valéry, Husserl, Éco, Proust, Stendhal, Paris : Maisonneuve et Larose, 2004.

Plotin, Ennéades III, (trad. E. Bréhier), Paris : Les Belles Lettres, 1995.

Prigogine, Ilya, Stengers, Isabelle, La nouvelle Alliance. Métamorphose de la science, Paris : Folio, 1986.

Schoenberg, Arnold, Traité d'harmonie, (Trad. G. Gubisch), Paris : Médiamusique, 2008.

Thom, René, Stabilité structurelle et morphogenèse, Paris : Interéditions, 1972.

- Esquisse d'une sémiophysique : Physique aristotélicienne et Théorie des Catastrophes, Paris : Interéditions, 1988.

Wittgenstein, Ludwig, Tractatus logico-philosophicus, (trad. G-G. Granger), Paris : Gallimard, 2001. 


\title{
RESUME
}

A la question de savoir si le formalisme contemporain parvient à restituer une vue suffisamment dynamique des lois naturelles, nous proposons une autre voie pour appréhender les mécanismes d'organisation des phénomènes naturels et sémiotiques. La percée des découvertes topologiques de René Thom permet la réhabilitation d'un cheminement philosophique, qui enregistre la fécondité d'un savoir qualitatif des formes dans leur déploiement. Notre étude souhaite soulever l'enjeu des interrogations suivantes : dans le sillage de Leibniz, quels échos attendre d'une posture néo-aristotélicienne visant à questionner objectivement l'organisation morphologique des phénomènes naturels ? Comment assumer la plasticité structurelle des épisodes de métamorphose en s'appuyant sur les prolongements actuels du seul mécanisme cartésien ? Quelle réhabilitation des formes substantielles pourrait nous aider pour penser aujourd'hui la nature?

Mots-clés : René Thom - morphologie - substance - Leibniz - Aristote

\begin{abstract}
To the issue whether the contemporary formalism could sufficiently provide a dynamical view of natural laws, we propose another way to understand the organizational mechanisms of natural and semiotic phenomena. The breakthrough of René Thom's topological discoveries allows the renewal of a philosophical path, which examines the benefits of a qualitative knowledge on forms through their deployment. Our study would like to raise the issue of the following questions: in the aftermath of Leibniz's work, which lessons can we expect to draw from a neo-Aristotelian position in order to objectively analyze the morphological organization of natural phenomena? How to consider dynamical plasticity from metamorphosis episodes, if we only take into account the contemporary development of the Cartesian mechanism? What rehabilitation of substantial forms might help to think about nature today?
\end{abstract}

Keywords: René Thom - Morphology - Substance - Leibniz -Aristotle 